Check for updates

Cite this: Anal. Methods, 2019, 11, 6023

\title{
Improved performance of near infrared excitation Raman spectroscopy using reflective thin-film gold on glass substrates for cytology samples
}

\author{
Sinead J. Barton, (D) a Kevin O'Dwyer, (D) a Marion Butler, ${ }^{\mathrm{b}}$ Adam Dignam, (D) ${ }^{\mathrm{b}}$ \\ Hugh J. Byrne, (D) c Luke O'Neill (iD c and Bryan M. Hennelly iD *ad
}

\begin{abstract}
Confocal near-infrared Raman spectroscopy has been shown to have applications in the area of clinical biology. A source wavelength in the near infrared is preferred over visible wavelengths for inspecting biological samples due to superior wavenumber resolution and reduced photodamage. However, these excitation sources have a number of drawbacks when compared to lasers in the visible wavelength region, including the requirement to use expensive highly pure crystal substrates such as Raman grade calcium fluoride as well as long acquisition times due to the lower Raman scattering efficiency. This paper investigates the use of a reflective substrate comprising a low cost $100 \mathrm{~nm}$ thin-film gold on glass substrate, as an alternative. Similar to recent work that used stainless steel substrates, it is demonstrated that the thin-film gold coated substrates, which are relatively inexpensive, produce cell spectra with 1.65 times the signal to noise ratio when compared with spectra obtained from calcium fluoride under identical conditions, with no apparent background signal in the fingerprint region. Two prostate cell lines are examined having been deposited on glass, calcium fluoride, and thin-film gold on glass substrates using the ThinPrep standard. Background spectra from, and cell adhesion on, these three substrates are compared. A comparison of the intensities and signal to noise ratios of the resulting spectra, and their viability for classification using principle components analysis is performed, which further demonstrates the benefit of reflective substrates.
\end{abstract}

Received 4th August 2019

Accepted 8th November 2019

DOI: 10.1039/c9ay01672d

rsc.li/methods

\section{Introduction}

Raman spectroscopy has been shown to have widespread application in the areas of clinical biology and medicine. ${ }^{\mathbf{1 - 4}}$ Confocal Raman microspectroscopy (RMS), in conjunction with multivariate classification, is a commonly applied technique for the biochemical analysis of tissues and cells. ${ }^{1-7}$ Typically, this involves first mounting the samples onto a substrate, which can be achieved either in vitro or ex vivo, followed by analysis with a microscope that has been integrated with a Raman spectroscopy system. ${ }^{8}$

Excitation of the Raman spectrum using a source laser with a wavelength in the near infrared (NIR) has a number of key advantages for analysing biological samples when compared with visible wavelength excitation: (i) firstly, photons in the NIR are absorbed to a lesser degree in tissues and cells and there is, therefore, less likelihood of burning or damaging the sample;

${ }^{a}$ Department of Electronic Engineering, Maynooth University, Maynooth, Co. Kildare, Ireland. E-mail: bryan.hennelly@mu.ie

${ }^{b}$ Department of Biology, Maynooth University, Maynooth, Co. Kildare, Ireland ${ }^{c}$ FOCAS Research Institute, TU Dublin, Kevin St, Dublin 8, Ireland

${ }^{d}$ Department of Computer Science, Maynooth University, Maynooth, Co. Kildare, Ireland (ii) secondly, biological samples are known to produce little or no auto-fluorescence with NIR lasers, the presence of which can be problematic when comparing spectra; and (iii) the wavenumber resolution is superior in a Raman spectrum scattered using a NIR wavelength (for example, the resolution at $785 \mathrm{~nm}$ is $\sim 2.3$ times smaller than that at $532 \mathrm{~nm}$ ).

However, despite the advantages discussed above, there also exist a number of disadvantages associated with NIR excitation: (i) firstly, and most significantly, the spectrum from a glass substrate has a large fluorescence that overwhelms the cell spectrum. This necessitates the use of expensive, highly pure Calcium Fluoride $\left(\mathrm{CaF}_{2}\right)$ substrates $(>\$ 100$ per slide) or similar. (ii) Secondly, the rate of Raman scattering is appreciably reduced (scales as a function of the fourth power of the inverse wavelength) for excitation using longer wavelengths, which results in lower values of the signal to noise ratio (SNR); for example, it can be shown that $532 \mathrm{~nm}$ excitation source produces Raman spectra that are 4.74 times more intense than those produced by a $785 \mathrm{~nm}$ source laser of equivalent power, assuming non-resonant conditions. There is, therefore, a requirement for increased acquisition times when using longer excitation wavelengths.

In recent years, the applications of RMS to biological cells for the purpose of diagnostics has increased, with many reporting the 
classification of bladder, ${ }^{9-13}$ cervical, ${ }^{14}$ and oral $^{15}$ cytological samples with a sensitivity and specificity that is greater than standard pathology. Due to the limitations of NIR excitation in terms of substrate cost, and long acquisition times, it is impractical for high throughput applications such as cytology for screening, the most obvious example being the Pap smear, and, therefore, excitation with lower wavelengths is typically preferred. Despite the advantages of Raman spectroscopy with NIR excitation, the high cost of highly pure Calcium Fluoride $\left(\mathrm{CaF}_{2}\right)$ substrates has limited the application of this modality in the areas of cytology and histopathology. In recent years, there is growing interest in alternative substrates that provide a low background spectrum and at low cost, particularly in the use of reflective metallic substrates, which also appear to enhance the Raman spectrum of samples deposited on the surface of these substrates.

Thin-film aluminium coated glass slides have been used as a substrate for biological samples in a number of studies involving Raman spectroscopy including for urine cytology, ${ }^{11}$ the study of lung cells ${ }^{16}$ and cervical tissue ${ }^{17}$ samples. An enhancement in the intensity of the Raman spectrum of epithelial cells deposited on reflective aluminium on glass substrates was first identified in a recent study that compared several different substrates with respect to their suitability for Raman spectroscopy with cytology samples using various excitation wavelengths from $405 \mathrm{~nm}$ up to $830 \mathrm{~nm}$. These substrates included glass, $\mathrm{CaF}_{2}$, fused silica, magnesium fluoride, and thin-film aluminium on glass as well as others. It was found that the aluminium coated slides had the lowest background signal of all the substrates measured including for NIR excitation wavelengths of $785 \mathrm{~nm}$ and $830 \mathrm{~nm}$. Based on the relatively intense Raman spectra recorded from cheek cells on these substrates, the authors suggested that the reflection provided by these substrates could provide up to four times more Raman scattered photons than transparent substrates due to a double pass of the laser through the sample as well as the collection of forward scattered photons. Following this work, inexpensive aluminium foil taped onto glass slides was investigated as a suitable substrate for the Raman analysis of histological and cytological samples. ${ }^{18,19}$

Mirrored stainless steel slides were subsequently investigated as suitable substrates for Raman spectroscopy with $785 \mathrm{~nm}$ excitation..$^{20}$ Formalin fixed paraffin embedded colon tissue blocks were mounted on stainless steel slides as well as on $\mathrm{CaF}_{2}$. In a separate experiment human bone osteosarcoma cells were cultured on both of these substrates. The authors demonstrated an improvement in the signal to noise ratio of samples recorded on steel compared with the same samples on $\mathrm{CaF}_{2}$; an improvement of 1.43 was reported for tissue samples and 1.64 for cells. In this paper, we perform a similar investigation for the case of prostate cancer epithelial cells deposited on $100 \mathrm{~nm}$ thin-film gold on glass and we report similar results with respect to the improvement in signal to noise ratio.

Other related work includes the demonstration of enhancements in Raman spectra for chemical samples placed on a reflective substrate ${ }^{21}$ and in ref. 22 it was shown that a reflective silver-coated substrate offered an enhancement of the signal from fluorescently labelled dog-kidney cells with an enhancement of $3-4$, which is similar to the level of enhancement of intensity determined in this paper with respect to the Raman spectrum. The authors in that paper propose that the same effect could be achieved with a thin-film gold substrate, with the additional benefit of having a high level of biocompatibility.

The purpose of this paper is to build on this previous work to investigate the potential of $100 \mathrm{~nm}$ thin-film gold on glass substrates for Raman cytology in conjunction with the ThinPrep slide preparation standard. All samples presented in this paper, regardless of substrate, were prepared using the ThinPrep standard, thus highlighting the applicability of this methodology in a clinical setting. We investigate the adhesion of cells to the surface of the gold using this standard and compare with $\mathrm{CaF}_{2}$ and glass. The background spectrum from the thin-film gold is also compared with $\mathrm{CaF}_{2}$ and glass and the enhancement of the signal to noise ratio of the cell spectrum on the thinfilm gold is investigated compared with cells on $\mathrm{CaF}_{2}$. Multivariate statistical classification of Raman spectra of two prostate cell lines is demonstrated using both $\mathrm{CaF}_{2}$ and thin-film gold on glass. The work is contextualised in terms of the recent progress in this area.

\section{Methods}

\subsection{Thin-film gold on glass substrates}

Thin-film gold on glass $(2.5 \mathrm{~cm} \times 7 \mathrm{~cm} \times 1 \mathrm{~mm})$ substrates were purchased from Deposition Research Laboratory Inc. at a cost of $\$ 5$ per slide. Prior to deposition, substrates were precleaned in vacuum using an ion source in a clean room setting. A titanium adhesion layer of $40 \mathrm{~nm}$ thickness is first deposited, followed by a gold layer of thickness $100 \mathrm{~nm}$. Films were deposited in a dedicated physical vapor deposition system using an electron-gun in high vacuum. Physical thickness was measured using on-site interferometer.

\subsection{Sample preparation}

High grade prostate cancer epithelium cells (PC3; SigmaAldrich) and androgen-sensitive human prostate adenocarcinoma (LNCaP, Sigma-Aldrich), were cultured in 1:1 mixture of DMEM and Hams-F12 medium supplemented with 5\% fetal bovine serum and $2 \mathrm{mM}$ LGlutamine. Flasks were maintained in a humidified atmosphere with $5 \% \mathrm{CO}_{2}$ at $37{ }^{\circ} \mathrm{C}$. When the cell lines reached $80 \%$ confluency, the culture medium was removed, and the cells were rinsed with sterile PBS. TrypsinEDTA $(0.5 \%)$ was added to the flask, which was incubated at $37^{\circ} \mathrm{C}$ until the cells had completely detached (not exceeding 15 min). An equal volume of $5 \%$ serum-containing medium was added to the flask to neutralise the trypsin enzyme. The entire contents of the flask was transferred into a sterile container, and centrifuged at $1200 \mathrm{rpm}$ for $5 \mathrm{~min}$. The supernatant was removed, and the cell pellet was resuspended in fresh medium. This solution was centrifuged at $1200 \mathrm{rpm}$ for $5 \mathrm{~min}$, the medium decanted, and resuspended in $1 \mathrm{ml}$ PBS. This step was repeated and the cell pellets were resuspended into a vial containing $20 \mathrm{ml}$ of a methanol based fixative (PreservCyt; Hologic, 
USA), and left at room temperature for $15 \mathrm{~min}$. The vial was inserted into a ThinPrep 2000 (T2; Hologic, USA) machine, and the cells were transferred on to either a gold coated glass slide (100 nm gold thin film on glass; Deposition Research Laboratory Inc., USA), a $\mathrm{CaF}_{2}$ (Raman Grade; Crystran, UK) slide, or a glass slide (ThinPrep slide; Hologic, USA). The ThinPrep standard has previously been shown to be compatible with Raman microspectroscopy. ${ }^{\mathbf{9}, 13}$ Images of the LNCaP cells on the three substrates are shown in Fig. 1. The image of the $\mathrm{CaF}_{2}$ and glass substrates was recorded using an Olympus IX81 inverted transmission microscope while the image of the gold substrate was recorded using a BX51 in reflection mode. The same camera (Amscope MU-300) was used to record all of the images shown in the figure and for all three substrates, the $4 \times$ and $50 \times$ images were recorded using Olympus PlanN $4 \times / 0.1$ and UMPlanFl $50 \times /$ 0.8 microscope objectives.

\subsection{Spectral acquisition}

All spectra were recorded using a HORIBA Jobin Yvon HR 800 (Villeneuve d'Ascq, France) Raman spectrometer, which was coupled to an Olympus BX41 upright microscope equipped with a $100 \times$ objective $($ MPlanN, Olympus, NA $=0.9)$ and a $785.16 \mathrm{~nm}$ diode laser source (300 $\mathrm{mW})$. Raman scattering was collected through a $400 \mu \mathrm{m}$ confocal hole onto a back illuminated aircooled CCD detector with $13.5 \mu \mathrm{m}$ pixel size (Synapse; Horiba, Villeneuve d'Ascq, France) for the range of 500-1800 $\mathrm{cm}^{-1}$ using a 300 lines per $\mathrm{mm}$ diffraction grating, yielding a dispersion of $\sim 1.5 \mathrm{~cm}^{-1}$ per CCD pixel. The instrument was calibrated using the $520 \mathrm{~cm}^{-1}$ silicon peak. All Raman spectra were recorded from single acquisitions of $20 \mathrm{~s}$ duration from a diffraction limited spot on the cell nucleus. Spectra were recorded from 52 PC3 cells on $\mathrm{CaF}_{2}, 52$ LNCap cells on $\mathrm{CaF}_{2}, 52$ PC3 cells on gold, and 52 LNCap cells on gold.

\subsection{Processing of Raman spectra}

Cosmic ray removal was implemented using the approach outlined in ref. 23 followed by application of an Extended Multiplicative Signal Correction (EMSC) algorithm. ${ }^{\mathbf{1 0 , 2 4}}$ This algorithm computes a background signal in the form of a baseline $N$ order polynomial (to remove the baseline signal that results from fluorescent signals or stray light due to Mie scattering ${ }^{25}$ ) plus the background signal from the substrate. Briefly described, the EMSC algorithm applies a least squares fit to (i) a reference Raman spectrum from a cell and (ii) an $N$ order polynomial. The algorithm returns the weight of (i), which enables normalisation of the spectrum relative to the reference, as well as the polynomial. The EMSC-corrected spectrum, $X$, is given by:

$$
X=\frac{X_{0}-\sum_{m=0}^{N} c_{m} P^{m}}{c_{\mathrm{r}}}
$$

where $X_{0}$ is the raw data, $P^{m}$ denotes the $m^{\text {th }}$ order of the polynomial, $c_{m}$ is the corresponding polynomial coefficient, and $c_{\mathrm{r}}$ is the weight of the cell reference spectrum, $R$. In summary, $X_{0}$ can be described as the linear (weighted) superposition of $R$ and $P$. It
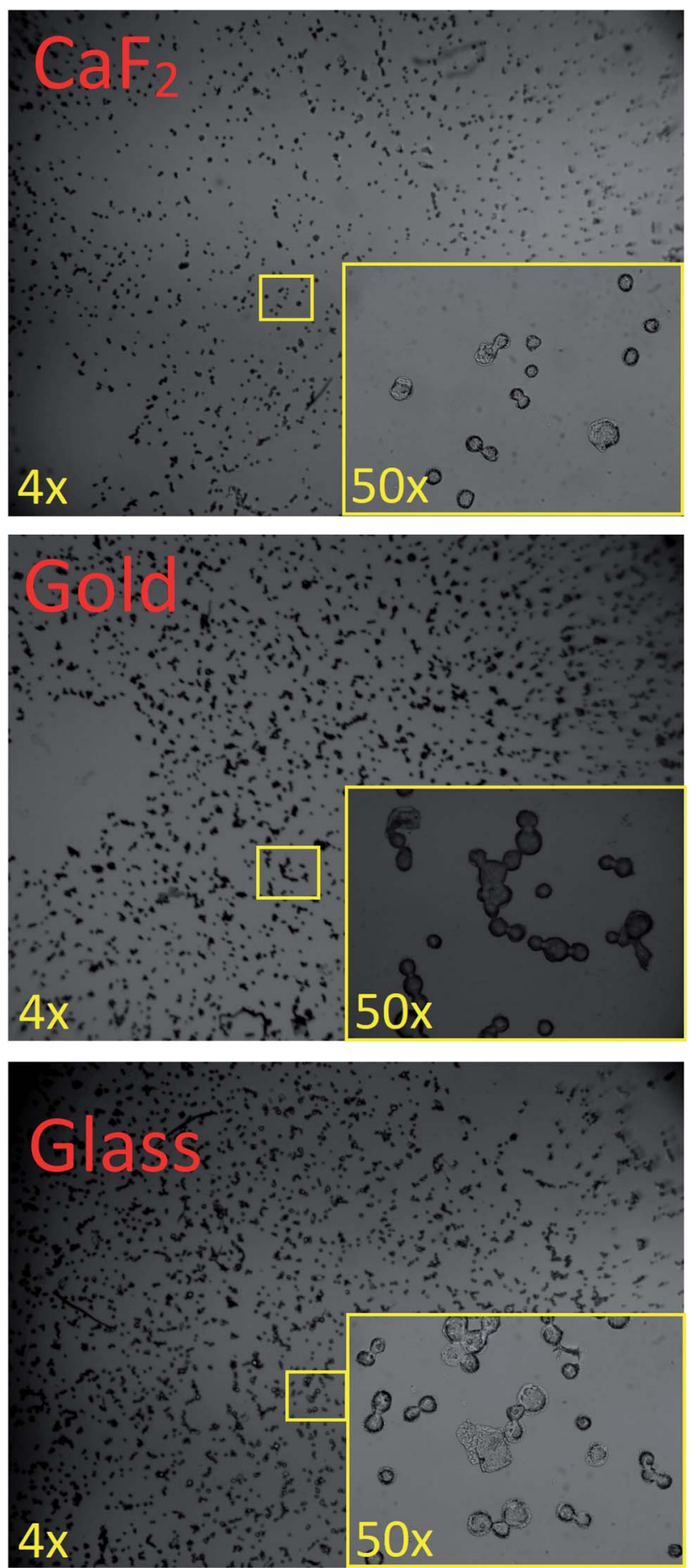

Fig. 1 Images of cells on substrates. Images recorded from a $\mathrm{CaF}_{2}$ slide, a $100 \mathrm{~nm}$ thin-film gold slide, and a glass ThinPrep slide. All three slides were prepared using the ThinPrep processor using the same concentration of LNCaP cells. The area of the $4 \times$ images is $8 \mathrm{~mm}^{2}$ and the area of the $50 \times$ images is $0.65 \mathrm{~mm}^{2}$.

has been shown that the use of a high-order polynomial does not result in over-fitting with the EMSC algorithm. ${ }^{26}$ For this study, a seventh order polynomial was used in the EMSC subtraction algorithm for all datasets, and similar results were found using lower orders. 
The reference cell spectrum provides the basis for all of the spectra to be fitted; the reference spectrum used here is the mean spectrum of all of the raw spectra recorded from all cells on both the thin-film gold on glass and $\mathrm{CaF}_{2}$ substrates. In order to remove any potential bias, the same reference spectrum was used for the EMSC algorithm applied to process the spectra of all cells that are investigated in this paper.

Multivariate statistical analysis was applied to the EMSC corrected Raman spectroscopic data for classification using Principle Components Analysis (PCA) and Linear Discriminant Analysis (LDA). PCA was directly applied, using the 'pca' function in MATLAB, to the four datasets recorded from cells on thin-film gold and $\mathrm{CaF}_{2}$ following the processing as described above. Subsequently, LDA was also applied using the 'fitcdiscr' function in MATLAB; the PCA scores accounting for $95 \%$ of the variance were included in the LDA and the performance of the classification was evaluated with Leave-One-Out (LOO) crossvalidation. In advance of PCA, all spectra were also smoothed with a Savitzky-Golay filter ${ }^{27}$ (polynomial order $=3$; window size $=11$ ) in order to reduce the effect of noise.

\subsection{Signal to noise ratio}

The SNR at a single point in the spectrum may be defined as follows: ${ }^{23,28,29}$

$$
\mathrm{SNR}=\frac{i t}{\sqrt{t(i+c)+r^{2}}}
$$

where $i$ represents the mean spectral irradiance at the sample point (i.e. on one pixel), $t$ is the acquisition time, $c$ is the mean rate of dark current generation (within the pixel), and $r$ is the standard deviation of the read noise. The irradiance and dark current are modelled by a Poisson distribution and the read noise is modelled by a Gaussian distribution. The values of $c$ and $r$ can be obtained from the CCD specification sheet. The definition in eqn (2) relates to the SNR at a single point in the spectrum. However, in an experimental context, an estimate for the mean SNR of the spectrum is more practical. For all of the experimental results that follow, the SNR is mathematically defined as follows:

$$
\mathrm{SNR}=\frac{S}{\sigma}
$$

where $S$ is the maximum value of $R$, as described in the previous section, and $\sigma$ is the standard deviation of the total noise in $X . \sigma$ is calculated from $X$ by subtracting a SG smoothed version (polynomial order $=3$, window size $=9$ ) of $X$ in order to isolate the noise signal. ${ }^{30}$ The value of $S$ is taken from the reference spectrum, $R$, rather than the corrected spectrum, $X$, in order to exclude the affect of noise from the measurement of $S$, which can distort the measurement of the SNR. The method to calculate SNR is illustrated in Fig. 2.

\section{Results}

\subsection{Cell adhesion using the ThinPrep processor}

In total 10 images at $4 \times$ magnification, similar to those shown in Fig. 1, were recorded at random positions on each of the three

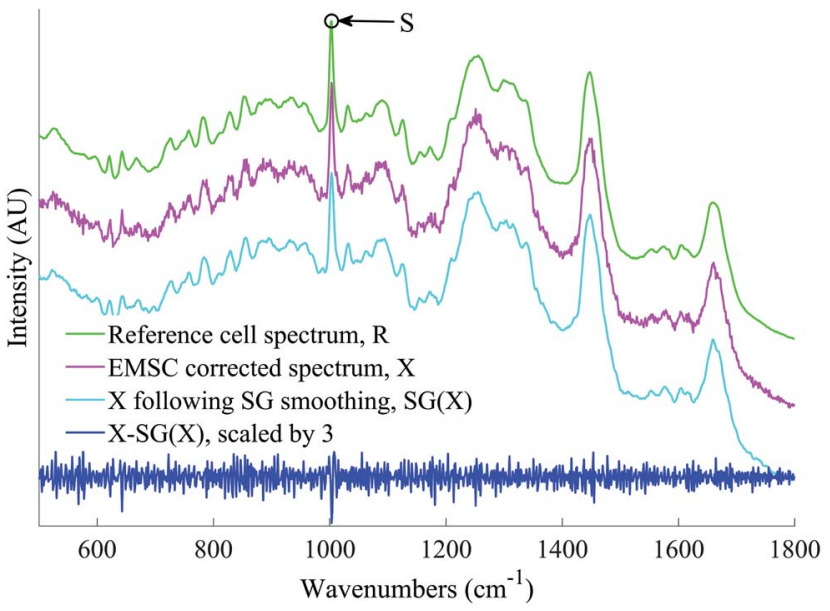

Fig. 2 Method for estimating the SNR of a spectrum. Spectra have been offset for clarity. $S$ is the maximum value in the reference cell spectrum.

slides and Image w was used to identify and count individual cells. The resulting average cell count and standard deviation on $\mathrm{CaF}_{2}$ were calculated to be 137.63 cells per $\mathrm{mm}^{2}$ and 70.90 cells per $\mathrm{mm}^{2}$. For glass these values were 213.47 cells per $\mathrm{mm}^{2}$ and 48.02 cells per $\mathrm{mm}^{2}$, and for thin-film gold on glass the values were 213.5 cells per $\mathrm{mm}^{2}$ and 16.98 cells per $\mathrm{mm}^{2}$.

\subsection{Background spectra from $100 \mathrm{~nm}$ thin-film gold on glass substrate}

In Fig. 3, spectra recorded from the $\mathrm{CaF}_{2}$, glass, and thin-film gold on glass substrates are shown; for all three cases, the laser was focused on the surface of the substrate to a diffraction limited spot using the system described in Section 2. In order to obtain a spectrum over a wider bandwidth from $200 \mathrm{~cm}^{-1}$ up to $3600^{-1}$, the grating was scanned, and multiple spectra were recorded and stitched together. The background spectrum is also shown, which is the result of recording a signal when the

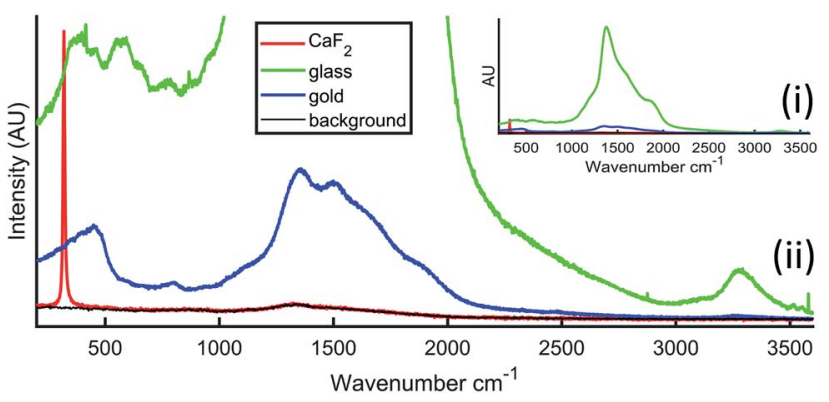

Fig. 3 Comparison of the spectra recorded from substrates; Raman grade $\mathrm{CaF}_{2}$ slide (red), ThinPrep glass slide (green), $100 \mathrm{~nm}$ thin-film gold on glass substrate (blue) and a background spectrum recorded when no sample was present (black). In all cases, the $785 \mathrm{~nm}$ wavelength laser was focused onto the surface of the substrate with a total acquisition time of $200 \mathrm{~s}$; (i) shows a sufficiently large range of intensity in order to fully capture the glass spectrum and (ii) shows a reduced ( 0.13 times) range in order to display more detail on the thin-film gold and $\mathrm{CaF}_{2}$ spectra. 
laser is active but no sample is present; this spectrum is made up of camera dark current, ambient light, and scattering from various optical elements in the system. Neglecting the variation in noise, this background will be present in the spectra recorded from the three substrates. For each of the four cases shown in the figure, 10 spectra were recorded with acquisition times of $20 \mathrm{~s}$ and averaged together. Other than cosmic ray removal no processing has been applied to the raw spectra.

\subsection{Classification of cell lines on different substrate}

The raw spectra recorded from the PC3 and LNCap cell lines on both thin-film gold on glass and $\mathrm{CaF}_{2}$ are shown in Fig. 4(i) and (ii) following cosmic ray removal. It should be noted that no scaling or intensity shift has been applied; the spectra shown in Fig. 4(i) and (ii) are shown on the same axis and with the same relative intensity as when they were recorded. On average, the spectral intensities of the cell spectra recorded from both cell lines on the thin-film gold is similar and is $\sim 3$ times that of the spectral intensities recorded on the $\mathrm{CaF}_{2}$. The corresponding datasets after EMSC correction are shown in Fig. 4(iii) and (iv). It is clear from these figures that there exists significantly less variance across the datasets recorded from the thin-film gold on glass substrate, particular in the wavenumber band around $900 \mathrm{~cm}^{-1}$ and in the band $1200-1400 \mathrm{~cm}^{-1}$. This is illustrated
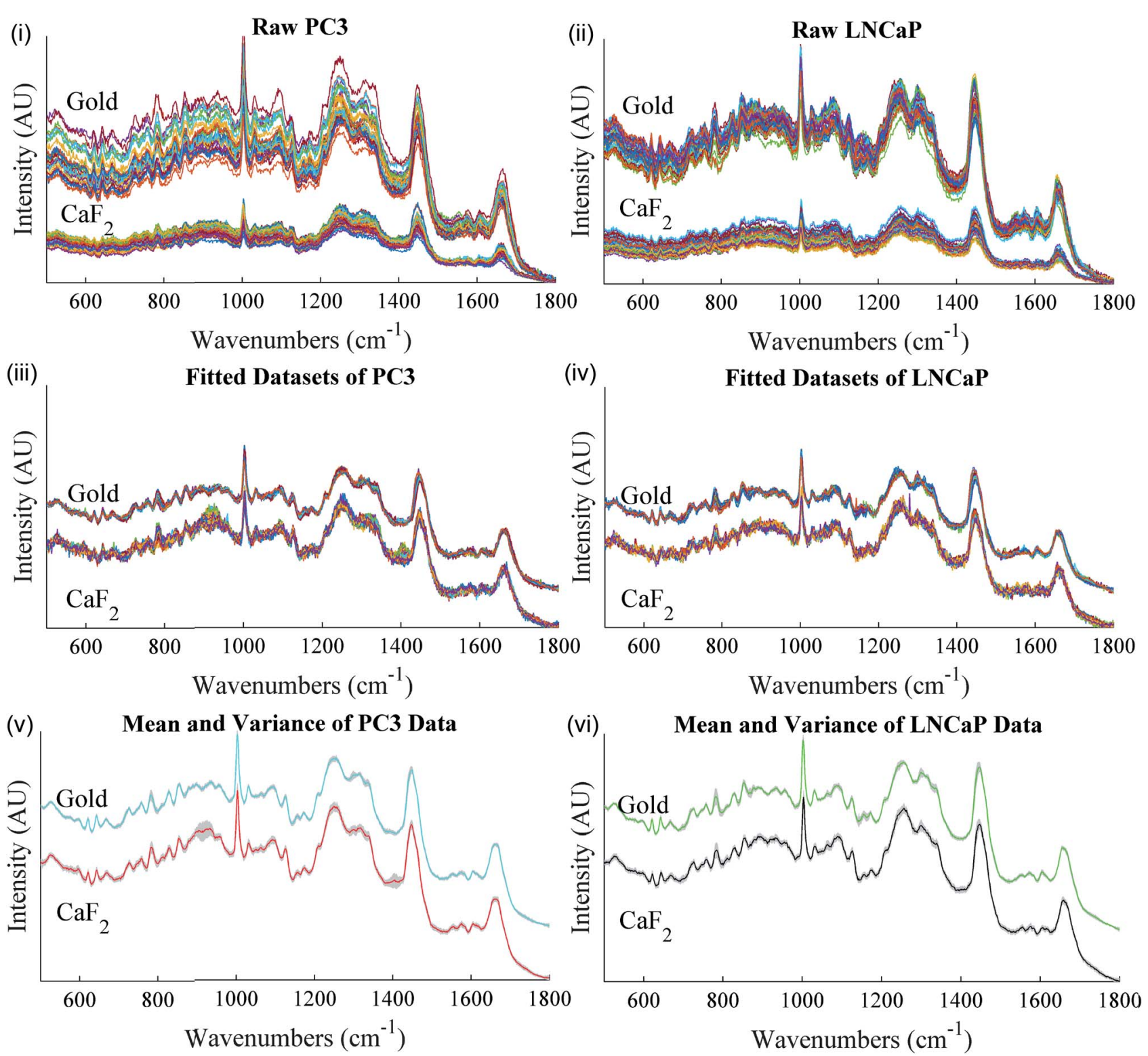

Fig. 4 Raw and processed spectral datasets recorded from two prostate cell lines on $\mathrm{CaF}_{2}$ and thin-film gold substrates. These figures illustrate the difference in spectral quality of cell spectra recorded from two cell lines on thin-film gold on glass and $\mathrm{CaF}_{2}$. The raw unscaled spectra are shown in (i) for cell line PC3 and (ii) for cell line LNCaP. Note, the intensity of the cells on thin-film gold on glass is $\sim 3$ times higher. In (iii) and (iv) the EMSC corrected datasets are shown and the two groups are shifted in the intensity axis for ease of comparison. In (v) and (vi) the mean spectrum and $\pm \sigma / 2$ of each sample point are shown for each of the four datasets shown in the previous two figures. 
more clearly in Fig. 4(v) and (vi) in which the mean spectra and standard deviation are shown for each of the EMSC corrected datasets. The difference in spectral intensity is further investigated by calculating the SNR of the spectra recorded from both substrates using the method described in Section 2.5. Although both datasets appear similar after normalisation, the spectra from cells on thin-film gold exhibit a higher SNR; the results are shown using a box and whisker plot in Fig. 5. The mean SNR for the PC3 and LNCaP cell spectra were 141.2 and 146.4 for the thin-film gold on glass substrate, respectively. The corresponding SNR values for the $\mathrm{CaF}_{2}$ substrate were 88.4 and 86.2. The thin-film gold substrate shows a consistently higher SNR than that of their $\mathrm{CaF}_{2}$ counterparts as well as a smaller standard deviation of SNR values.

PCA is applied in order to investigate (i) the variation between the spectra recorded across both cell lines for each substrate type and (ii) the difference in the separation of the same cell line across the two substrates individually. The results are shown in Fig. 6 and 7, in which the same colour codes for the four cell groups as have appeared elsewhere in this paper. For each case, only the principal components (PrC) are shown for which there is a clear separation of the scores. In Fig. 6(i), the mean spectra of PC3 and LNCaP deposited on thin-film gold on glass are shown as well as the second principal component, PrC2 following PCA of these two datasets. PrC1, PrC2, and PrC3 account for $56.3 \%, 7 \%$, and $3.4 \%$ of the variance, respectively, and PrC2 primarily accounts for the separation between the two datasets; (ii) shows the corresponding scatter plot. Corresponding results are shown for both cell lines when deposited on $\mathrm{CaF}_{2}$ in Fig. 6(iii) and (iv). In this case PrC1, PrC2, and PrC3 account for $26.5 \%, 16.1 \%$, and $5.2 \%$ of the variance, respectively and the scores are separated in both PrC1 and PrC2. Prominent peaks in the relevant loading(s) have been highlighted for both cases and a close correlation is observed in the peak regions

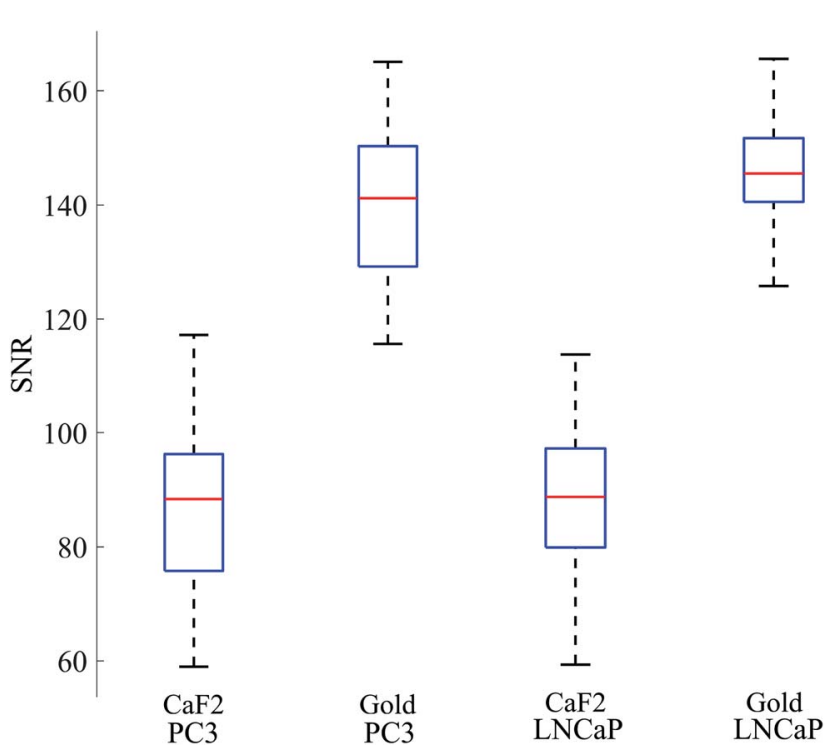

Fig. 5 A comparative boxplot of SNR values for both cell lines across $\mathrm{CaF}_{2}$ and $100 \mathrm{~nm}$ thin-film gold on glass substrates. The average value of enhancement provided by the reflective thin-film substrates is 1.65 .
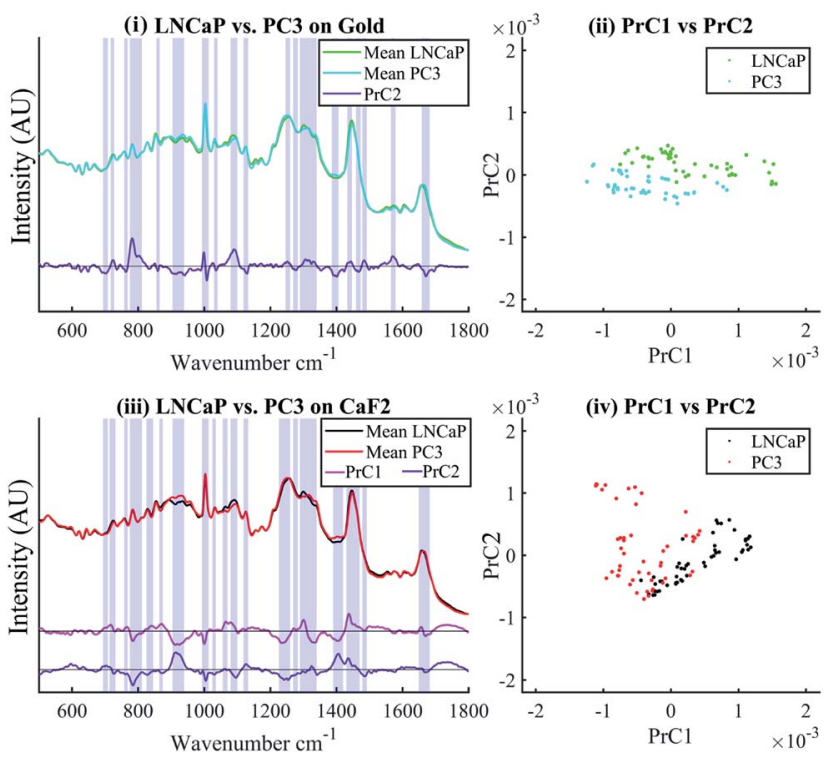

Fig. 6 PCA analysis of two cell lines for the case of $\mathrm{CaF}_{2}$ and separately for the case of thin-film gold substrates. Separation is observed with either substrates, based on similar spectral differences. (i) Mean LNCaP and PC3 cell spectra on thin-film gold on glass as well as the PCA loading providing maximum separation; (ii) corresponding scatter plot; (iii) mean spectra of $\mathrm{LNCaP}$ and $\mathrm{PC} 3$ deposited on $\mathrm{CaF}_{2}$ as well as the two PCA loadings that provide maximum separation; (iv) corresponding scatter plot.

that are relevant to classification. These include regions around numerous spectral peaks that have previously been noted in the classification of prostate and bladder cells, including 792
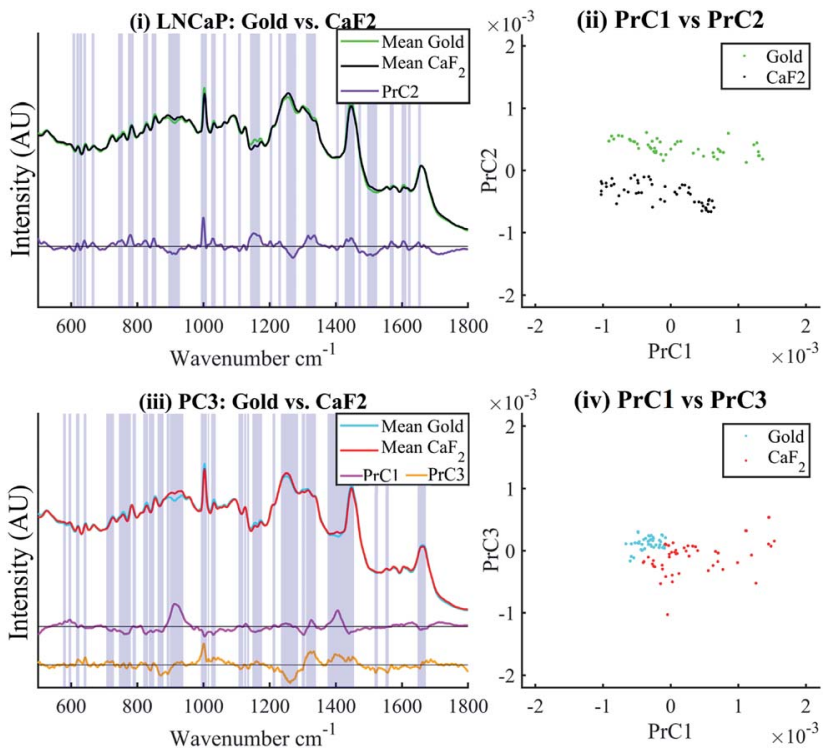

Fig. 7 PCA analysis to investigate why spectra of the same cell line are different when recorded from $\mathrm{CaF}_{2}$ and thin-film gold substrates. (i) Mean $\mathrm{LNCaP}$ cell spectra recorded on thin-film gold on glass and $\mathrm{CaF}_{2}$ as well as the PCA loading providing maximum separation between these two datasets; (ii) corresponding scatter plot; (iii) mean spectra of and PC 3 cells deposited on thin-film gold on glass and $\mathrm{CaF}_{2}$ as well as the PCA loading that provides maximum separation; (iv) corresponding scatter plot. 
(DNA), 938 (proteins), 1002 (phenylalanine; protein), 1058 (DNA, lipids), 1089 (DNA), 1252 (amide III), $1302\left(\mathrm{CH}_{2}\right.$; lipids) $1340\left(\mathrm{CH}_{2} / \mathrm{CH}_{3}\right.$ wagging of nucleic acids), 1459 (DNA), 14841574 (DNA), and 1676 (amide I) $\mathrm{cm}^{-1} \cdot \mathbf{9 , 1 0 , 3 0 , 3 1}$ LDA was applied to the PC scores accounting for $95 \%$ of the variance and evaluated using a LOO cross validation. For the case of the thin-film gold on glass substrate, 38 principal components were included in the analysis and the sensitivity and specificity were both $98 \%$; for the $\mathrm{CaF}_{2}, 64$ principal components were included in the analysis and the sensitivity and specificity were $98 \%$ and $96 \%$, respectively.

In order to further investigate the effect of the substrates, PCA was also applied to examine the variation in spectra recorded from the same cell line across the two substrate types; these results are presented in Fig. 7. For the case of LNCaP cell spectra, PrC1, PrC2, and PrC3 account for 34.6\%, 14.5\%, and $5.2 \%$ of the variance, respectively and for the PC3 cell spectra, PrC1, PrC2, and PrC3 account for $25.8 \%, 19.7 \%$, and $6.6 \%$ of the variance, respectively. Although the scores are clearly separating for both sets of analysis, there appears to be no obvious relationship in the loadings that relate to this separation.

\section{Discussion}

\subsection{Cell adhesion using the ThinPrep processor}

The ThinPrep processor employs the use of filter membranes that collect the cells on their surface following a vacuum operation. The membrane is subsequently inverted and gently pressed against the substrate. The $100 \mathrm{~nm}$ thin-film gold and glass substrates were found to provide very similar levels of cell adhesion, while the $\mathrm{CaF}_{2}$ provided significantly less adhesion when used with the ThinPrep processor indicating that the surface properties of the gold are more suitable for cellular adhesion using the ThinPrep standard. Further work is required to investigate if other reflective substrates such as stainless steel $^{20}$ can achieve similar results in this regard. It should be noted that not all reflective substrates are as chemically stable as the thin-film gold on glass substrates used in this paper. In the course of this work, aluminium slides (100 $\mathrm{nm}$ Al deposited on glass) were investigated but were found to be reactive with the methanol fixative (Preservcyt) used to preserve the cells, which is supplied as a consumable to be used with the ThinPrep protocol.

\subsection{Background spectra from thin-film gold on glass substrate}

Referring to Fig. 3, the spectrum of the $\mathrm{CaF}_{2}$ substrate has a prominent sharp peak at $321 \mathrm{~cm}^{-1}$ and at other wavenumbers the intensity is approximately equal to that of the background. In contrast, the glass slide has an intense spectrum, particularly in the band between $1000-2000 \mathrm{~cm}^{-1}$. This spectrum has been attributed to the presence of active luminescent ion impurities in glass ${ }^{32}$ and has been shown to vary in profile and to increase in intensity as the source laser moves from the blue region up to the NIR region, ${ }^{7}$ making a laser in the lower end of the visible spectrum more desirable for Raman spectroscopy applied to clinical cytology samples mounted on glass. ${ }^{9}$
The spectrum recorded from the gold thin-film surface is also shown in Fig. 3. Although significantly weaker than the glass spectrum (the maximum values differ by a factor of 0.12 ), the spectrum is appreciably stronger than the spectrum of the $\mathrm{CaF}_{2}$. It has been shown that the transmittance of gold films of $100 \mathrm{~nm}$ thickness is close to zero ${ }^{33,34}$ and, therefore, it is likely that this spectrum originates primarily from the gold layer, and not from the titanium or glass. The physical process that generates the spectrum recorded from the gold has not been determined but it was possible to confirm that the profile of the spectrum changes when a $532 \mathrm{~nm}$ laser source was focused on the surface (not shown here). At the time of writing it was not possible to determine the purity of the gold used in the manufacture of the thin-film gold slides. It may be possible that similar to the case of glass, impurities in the gold contribute to a wavelength dependent photoluminescence spectrum but further work is required to confirm this.

Although the background spectrum from the gold film is appreciably large when compared with the spectrum recorded form the $\mathrm{CaF}_{2}$ substrate, features of the gold spectrum do not appear to be present in the spectra recorded from cells deposited on the gold surface shown in Fig. 4. Typically, when recording Raman spectra from a substrate that generates a background spectrum, this background spectrum will appear in varying amounts in the recorded spectra; see ref. 10 for an example of the glass background spectrum contaminating cell Raman spectra in varying amounts using $532 \mathrm{~nm}$ excitation. In such cases, the variance of the spectral dataset is relatively large in areas in which the background spectrum is more intense; this is not found to be the case here. Furthermore, there is no clear evidence of features of the profile of the gold background spectrum appearing in the loadings of the PCA analysis presented in Fig. 7, in which the same cell lines on $\mathrm{CaF}_{2}$ and thinfilm gold on glass were directly compared. The spreading out of the laser at the gold surface (during recording of a cell spectrum, the laser is focused on the cell nucleus at a point that is at least $1 \mu \mathrm{m}$ from gold surface), the short penetration depth of light in gold, ${ }^{33}$ and the effect of the confocal aperture ${ }^{35}$ all appear to reduce the presence of background spectrum from the gold in the Raman spectrum of the cell.

Another point of note relates to the reflectivity of gold as a function of wavelength. It has previously been shown that the reflectivity of gold in the NIR is $>0.95$ but that this drops rapidly over the visible region to $<0.3$ in the UV. ${ }^{33}$ It is likely, therefore, that the enhancement in Raman intensity that is reported in this study may not be reproducible for Raman spectroscopy with lower wavelengths. More work is also needed to fully characterise the background spectrum from the gold at other wavelengths.

\subsection{Enhancement of the signal-to-noise ratio}

There are a number of interesting observations that can be made from the SNR analysis presented in Fig. 5. Most notably, the mean SNR value of all the spectra recorded from cells on thin-film gold on glass is 143.8 and the mean SNR from cells on $\mathrm{CaF}_{2}$ is 87.3. The ratio of these values is 1.65 , which is 
approximately the square root of the ratio of the mean intensities of the raw spectral data recorded from both substrates, shown in Fig. 4(i) and (ii). This result is roughly in agreement with what would be expected given the square root relationship between the intensity and shot noise, taking into account the relatively low values of $c$ and $r$. Remarkably, this result is almost identical to the value of 1.64 times enhancement in SNR that was reported for a similar investigation using cells grown on reflective stainless steel substrate and $\mathrm{CaF}_{2} \cdot{ }^{20}$ It is likely that the enhancement provided by other reflective substrates will be similar to those reported from thin-film gold and stainless steel provided that the reflectivity of the metal is high over the wavelength range of interest.

The model that has been proposed for this enhancement $t^{7,20}$ is that the reflective substrate allows for forward scattered Raman photons, which would be lost through a transparent substrate, to be reflected back towards the microscope objective and collected resulting in an approximate doubling in Raman intensity. Secondly, the source laser is also reflected backwards, effectively doubling the excitation power in the sample. Therefore, for thin samples, reflective substrates will yield a maximum of quadruple the spectral intensity, compared with those recorded from samples deposited on a transparent substrate, resulting in an approximate doubling of the SNR as a direct consequence. For thicker samples, the focal point of the laser must be positioned further from the substrate surface and it can, therefore, be expected that the spectral intensity will increase by a factor of up to 4 and the increase in SNR will be up to a factor of 2 .

\subsection{Classification of cell lines on different substrate}

It can be inferred from PCA results that shown in Fig. 6 that approximately the same biomolecular differences account for the separation of the two prostate cell lines regardless of substrate. Although there are clear similarities in the principal components for both cases, there are also clear differences. It is difficult to account for the precise reasons for these differences since spectra recorded from the same two cell lines (taken from the same batch and recorded on the same day) have been compared for both cases. Given that the surface properties appear to affect the adhesion of the cells in different ways, it is possible that the surface properties also affect the Raman spectra that are recorded from the cells. The second PCA analysis shown in Fig. 7 attempted to elucidate the differences in spectra recorded from the same cell line across the two substrates; however, this analysis did not reveal any commonality in the features causing separation for the two cases. It is possible that the substrate surfaces affect the individual cell lines in different ways. More work is required to fully understand this phenomenon.

\subsection{Biocompatibility}

This study focuses on the use of gold coated substrates with fixed cells deposited using a commercial device for the preparation of cytology samples. Therefore, cytotoxicity issues associated with the surface properties of the gold were not considered. An active area of research is the interrogation of living cells using Raman spectroscopy, which typically involves growing cells directly onto a surface followed by Raman spectroscopy. Substrate surface chemical reactivity is of major importance if cells are to be grown on the substrate and although not investigated here, thin-film gold substrates may also be useful for Raman studies involving cell growth. Gold coated substrates such as those investigated in this study are known to be compatible with live cell growth, which often involves the functionalisation of the gold surface using monolayers of biochemicals that can be investigated for the capacity to promote or inhibit cell growth. ${ }^{36-38}$ Similar thin-film gold substrates as those used in this study have also previously been in the analysis of surface plasmon resonance for the real-time observation of biomolecular interactions, which involves the direct adhesion of the cells to the surface of the gold..$^{39,40} \mathrm{We}$ are not aware of any reports that coated gold substrates inhibit cell growth. Although, in recent work osteosarcoma cells were grown on stainless steel substrates for analysis with Raman spectroscopy, ${ }^{20}$ thin-film gold substrates may be preferable in terms of biocompatibility, with stainless steel having been shown to negatively impact on cell growth in some cases. ${ }^{\mathbf{4 1 , 4 2}}$

\section{Conclusion}

The first conclusion from this paper is that a $100 \mathrm{~nm}$ thin film of gold on glass substrate provides superior performance when compared with $\mathrm{CaF}_{2}$ for cytological samples prepared with the ThinPrep standard and analysed using Raman microspectroscopy. The thin-film gold spectra performed $54 \%$ better than the $\mathrm{CaF}_{2}$ with respect to cell adhesion and showed similar levels of adhesion compared with ThinPrep glass slides. It was also demonstrated that the thin-film gold substrates yield spectra with approximately three times the spectral intensity and 1.65 times the SNR when compared with $\mathrm{CaF}_{2}$. A relatively low intensity photoluminescence spectrum was recorded from the thin-film of gold but this does not appear to contaminate the cell spectra recorded from its surface; no evidence of this background spectrum could be found in subsequent multivariate statistical analysis. The gold substrate is significantly less expensive than Raman grade $\mathrm{CaF}_{2}, € 5$ per substrate compared with $€ 100$.

Principal components analysis was applied to spectra recorded from two prostate cancer cell lines, which broadly identified differences in the same set of peak locations regardless of substrate type, many of which have previously been highlighted in studies relating to bladder and prostate cancer. The results also demonstrate that the lower noise spectra recorded from the gold substrates provided for significantly better clustering of the data using PCA. We note, however, that some spectral differences were observed across the two substrates for the same cell lines; these differences do not appear to be consistent for the two cell lines investigated here; more work is required in order to understand this phenomenon.

Remarkably, the results presented here with respect to the enhancement of the signal to noise ratio of Raman spectra are 
almost identical to those reported in a related study using reflective stainless steel substrates. ${ }^{20}$ That study reported an enhancement of 1.64 for spectra of osteo cells grown directly on the substrate, compared with a value of 1.65 reported here for prostate epithelial cells deposited onto the surface using the ThinPrep standard. It may be inferred, therefore, that any suitably reflective substrate will provide three fold increase in Raman intensity, which will likely translate to a $\sim 66 \%$ reduction in acquisition time for equivalent spectral quality, which may be a significant consideration for some applications that require a large number of repeated measurements, such as the application of Raman cytology for diagnostics or mapping experiments. However, it is likely that different reflective substrates using various metals will have different surface properties that will affect cell adhesion or compatibility.

Reflecting substrates appear to offer an advantage for Raman cytology with NIR excitation when compared $\mathrm{CaF}_{2}$ in terms of both performance and cost. The optimal type of reflective substrate remains an open question and a subject for future research. More work is required to investigate other types of reflective thin-film metallic deposition on glass, and to evaluate all of the possible substrates in terms of cost, biocompatibility, cell adhesion, and compatibility with related chemicals.

\section{Conflicts of interest}

There are no conflicts to declare.

\section{Acknowledgements}

This research was conducted with the financial support of the Irish Research Council (IRC) under project ID GOIPG/2013/1434 and Science Foundation Ireland (SFI) under Grant Number 15/ CDA/3667. I would like to thank the IRC and SFI for their support.

\section{References}

1 C. Krafft, I. Schie, T. Meyer, M. Schmitt and J. Popp, Chem. Soc. Rev., 2016, 45, 1819-1849.

2 C. Krafft, M. Schmitt, I. W. Schie, D. Cialla-May, C. Matthäus, T. Bocklitz and J. Popp, Angew. Chem., Int. Ed., 2017, 56, 4392-4430.

3 K. Kong, C. Kendall, N. Stone and I. Notingher, Adv. Drug Delivery Rev., 2015, 89, 121-134.

4 H. J. Butler, L. Ashton, B. Bird, G. Cinque, K. Curtis, J. Dorney, K. Esmonde-White, N. J. Fullwood, B. Gardner, P. L. Martin-Hirsch, et al., Nat. Protoc., 2016, 11, 664.

5 J. Hutchings, C. Kendall, B. Smith, N. Shepherd, H. Barr and N. Stone, J. Biophotonics, 2009, 2, 91-103.

6 G. Clemens, J. R. Hands, K. M. Dorling and M. J. Baker, Analyst, 2014, 139, 4411-4444.

7 L. T. Kerr, H. J. Byrne and B. M. Hennelly, Anal. Methods, 2015, 7, 5041-5052.

8 R. Kiselev, I. W. Schie, S. Aškrabić, C. Krafft and J. Popp, Biomed. Spectrosc. Imaging, 2016, 5, 115-127.
9 L. T. Kerr, T. M. Lynn, I. M. Cullen, P. J. Daly, N. Shah, S. O'Dea, A. Malkin and B. M. Hennelly, Anal. Methods, 2016, 8, 4991-5000.

10 L. Kerr and B. Hennelly, Chemom. Intell. Lab. Syst., 2016, 158, 61-68.

11 A. Shapiro, O. N. Gofrit, G. Pizov, J. K. Cohen and J. Maier, Eur. Urol., 2011, 59, 106-112.

12 L. T. Kerr, K. Domijan, I. Cullen and B. M. Hennelly, Photonics Lasers Med., 2014, 3, 193-224.

13 F. Bonnier, D. Traynor, P. Kearney, C. Clarke, P. Knief, C. Martin, J. J. O'Leary, H. J. Byrne and F. Lyng, Anal. Methods, 2014, 6, 7831-7841.

14 L. F. C. Carvalho, F. Bonnier, K. O'Callaghan, J. O'Sullivan, S. Flint, H. J. Byrne and F. M. Lyng, Exp. Mol. Pathol., 2015, 98, 502-509.

15 A. F. Palonpon, J. Ando, H. Yamakoshi, K. Dodo, M. Sodeoka, S. Kawata and K. Fujita, Nat. Protoc., 2013, 8, 677.

16 J. K. Pijanka, N. Stone, A. V. Rutter, N. Forsyth, G. D. Sockalingum, Y. Yang and J. Sulé-Suso, Analyst, 2013, 138, 5052-5058.

17 L. E. Kamemoto, A. K. Misra, S. K. Sharma, M. T. Goodman, H. Luk, A. C. Dykes and T. Acosta, Appl. Spectrosc., 2010, 64, 255-261.

18 L. Cui, H. J. Butler, P. L. Martin-Hirsch and F. L. Martin, Anal. Methods, 2016, 8, 481-487.

19 M. Paraskevaidi, C. L. Morais, O. Raglan, K. M. Lima, E. Paraskevaidis, P. L. Martin-Hirsch, M. Kyrgiou and F. L. Martin, J. Biophotonics, 2018, e201700372.

20 A. T. Lewis, R. Gaifulina, M. Isabelle, J. Dorney, M. L. Woods, G. R. Lloyd, K. Lau, M. Rodriguez-Justo, C. Kendall, N. Stone, et al., J. Raman Spectrosc., 2017, 48, 119-125.

21 S. Lohumi, M. S. Kim, J. Qin and B.-K. Cho, Sensors, 2019, 19, 2698.

22 E. Le Moal, E. Fort, S. Lévêque-Fort, F. Cordelières, M.-P. Fontaine-Aupart and C. Ricolleau, Biophys. J., 2007, 92, 2150-2161.

23 S. J. Barton, T. E. Ward and B. M. Hennelly, Anal. Methods, 2018, 10, 3759-3769.

24 J. Skogholt, K. H. Liland and U. G. Indahl, J. Raman Spectrosc., 2019, 50(3), 407-417.

25 F. Bonnier, A. Mehmood, P. Knief, A. Meade, W. Hornebeck, H. Lambkin, K. Flynn, V. McDonagh, C. Healy, T. Lee, et al., J. Raman Spectrosc., 2011, 42, 888-896.

26 K. H. Liland, T. Almöy and B.-H. Mevik, Appl. Spectrosc., 2010, 64, 1007-1016.

27 A. Savitsky and M. Golay, Anal. Chem., 1964, 36, 1627-1639. 28 S. Barton and B. Hennelly, Biophotonics: Photonic Solutions for Better Health Care VI, 2018, p. 106854F.

29 S. J. Barton, L. T. Kerr, K. Domijan and B. M. Hennelly, Biophotonics: Photonic Solutions for Better Health Care V, 2016, p. 98873A.

30 T. J. Harvey, C. Hughes, A. D. Ward, E. C. Faria, A. Henderson, N. W. Clarke, M. D. Brown, R. D. Snook and P. Gardner, J. Biophotonics, 2009, 2, 47-69.

31 R. O. Draga, M. C. Grimbergen, P. L. Vijverberg, C. F. v. Swol, T. G. Jonges, J. A. Kummer and J. Ruud Bosch, Anal. Chem., 2010, 82, 5993-5999. 
32 D. Ehrt, IOP Conference Series: Materials Science and Engineering, 2009, p. 012001.

33 O. Loebich, Gold Bull., 1972, 5(1), 2-10.

34 A. Axelevitch, B. Apter and G. Golan, Opt. Express, 2013, 21, 4126-4138.

35 Z. Tang, S. J. Barton, T. E. Ward, J. P. Lowry, M. M. Doran, H. J. Byrne and B. M. Hennelly, Appl. Opt., 2018, 57, E118E130.

36 C. D. Tidwell, S. I. Ertel, B. D. Ratner, B. J. Tarasevich, S. Atre and D. L. Allara, Langmuir, 1997, 13, 3404-3413.

37 M. Mrksich, C. S. Chen, Y. Xia, L. E. Dike, D. E. Ingber and G. M. Whitesides, Proc. Natl. Acad. Sci. U. S. A., 1996, 93, 10775-10778.
38 L. H. Jin, B. Y. Yang, L. Zhang, P. L. Lin, C. Cui and J. Tang, Langmuir, 2009, 25, 5380-5383.

39 Y. Yanase, T. Hiragun, S. Kaneko, H. J. Gould, M. W. Greaves and M. Hide, Biosens. Bioelectron., 2010, 26, 674-681.

40 Y.-C. Kwon, M.-G. Kim, E.-M. Kim, Y.-B. Shin, S.-K. Lee, S. D. Lee, M.-J. Cho and H.-S. Ro, Biotechnol. Lett., 2011, 33, 921-927.

41 P. P. Vicario, Z. Lu, I. Grigorian, Z. Wang and T. Schottman, J. Biomed. Mater. Res., Part B, 2009, 89, 114-121.

42 A. Yamamoto, Y. Kohyama, D. Kuroda and T. Hanawa, Mater. Sci. Eng., C, 2004, 24, 737-743. 